Article

\title{
Integrated Metabolomics and Transcriptomics Unravel the Metabolic Pathway Variations for Different Sized Beech Mushrooms
}

\author{
Su Young Son ${ }^{1}$, Yu Jin Park ${ }^{1}$, Eun Sung Jung ${ }^{2}$, Digar Singh ${ }^{1}$, Young Wook Lee ${ }^{3}$, \\ Jeong-Gu Kim ${ }^{4, *}$ and Choong Hwan Lee ${ }^{1,2,5, *}$ \\ 1 Department of Bioscience and Biotechnology, Konkuk University, Seoul 05029, Korea; \\ syson119@naver.com (S.Y.S.); y_zing@naver.com (Y.J.P.); singhdigar@gmail.com (D.S.) \\ 2 Department of Systems Biotechnology, Konkuk University, Seoul 05029, Korea; jes708@naver.com \\ 3 Bumwoo Mushroom Farm Co. Ltd., 538 Yeoyang 1-ro, Yeoju 12609, Korea; bumwoo0789@naver.com \\ 4 Genomics Division, National Academy of Agricultural Science, Rural Development Administration, \\ Jeonju 54874, Korea \\ 5 Research Institute for Bioactive-Metabolome Network, Konkuk University, Seoul 05029, Korea \\ * Correspondence: jkim5aug@korea.kr (J.-G.K.); chlee123@konkuk.ac.kr (C.H.L.); \\ Tel.: +82-63-238-45-66 (J.-G.K.); +82-2-2049-6177 (C.H.L.)
}

Received: 12 October 2019; Accepted: 26 November 2019; Published: 28 November 2019

\begin{abstract}
Beech mushrooms (Hypsizygus marmoreus) are largely relished for their characteristic earthy flavor, chewy-texture, and gustatory and nutritional properties in East Asian societies. Intriguingly, the aforementioned properties of beech mushroom can be subsumed under its elusive metabolome and subtle transcriptome regulating its various stages of growth and development. Herein, we carried out an integrated metabolomic and transcriptomic profiling for different sized beech mushrooms across spatial components (cap and stipe) to delineate their signature pathways. We observed that metabolite profiles and differentially expressed gene (DEGs) displayed marked synergy for specific signature pathways according to mushroom sizes. Notably, the amino acid, nucleotide, and terpenoid metabolism-related metabolites and genes were more abundant in small-sized mushrooms. On the other hand, the relative levels of carbohydrates and TCA intermediate metabolites as well as corresponding genes were linearly increased with mushroom size. However, the composition of flavor-related metabolites was varying in different sized beech mushrooms. Our study explores the signature pathways associated with growth, development, nutritional, functional and organoleptic properties of different sized beech mushrooms.
\end{abstract}

Keywords: beech mushroom; different sizes; metabolomics; transcriptomics; metabolic pathway

\section{Introduction}

Mushrooms have traditionally been revered for their distinctive umami taste, flavors (earthy, meaty, or woodsy), and chewy texture coupled with varying nutraceutical functions [1]. Nutritionally, mushrooms are the quality source of proteins, polysaccharides, dietary fibers, vitamins, and minerals [2]. Beech mushrooms (Hypsizygus marmoreus) are native to East Asia and used in various culinary gourmets involving the 'Shimeji' mushroom groups [3]. In recent years, the applications of various strain improvement and cultivation methods have considerably improved the organoleptic properties of beech mushrooms attaining its ameliorated bitter taste, unique crunchy texture, and mild nutty flavor in a short cultivation period $[4,5]$. Beech mushrooms are rich in natural antioxidants and characteristic bioactive compounds, especially the hypsiziprenols having in vitro anti-proliferative effects on renal cancer cell lines $[3,6]$. Beech mushrooms have been increasingly recognized for their 
unique organoleptic, nutritional, and health promoting properties, however, the molecular mechanisms maneuvering the spatial distribution of nutrients and functional bio-actives across the fruiting body are largely unknown.

Mass spectrometry (MS)-based metabolomics approaches are widely used to study the metabolite profiles indicative of the key biosynthetic pathways governing the temporal growth and development of plants [7-9]. However, a limited number of metabolomics studies have reported the metabolic disparity among mushrooms across spatial dimensions and cultivars $[3,10]$. An approach utilizing integrated metabolomics and transcriptomics could provide a more powerful tool to understand the intertwined metabolism and molecular mechanisms of gene regulation through the construction of a single pathway [11-14]. Indeed, a number of studies have examined various microbes and plants, including Arabidopsis, Salvia, and Penicillium species using an integrated metabolomics-transcriptomics approach to reveal mechanisms underlying the biosynthesis of functional bio-actives including flavonoids, polyketides, and terpenoids [11-14].

Previously, we have reported the subtle variations in metabolite profiles of white and brown beech mushrooms across the stipe and cap portions which may help in mushroom selection and strain improvement [3]. In an extension to our previous study, we examined the metabolomic disparity among the beech mushroom groups of three different sizes (small, medium, and large) through the systematic construction of an integrated metabolic pathway derived from untargeted metabolite profiles and corresponding gene expression data. In the present study, we argue that different sized mushrooms may have markedly varying nutritional and functional values maneuvered by their distinct metabolomes, biosynthetic pathways, and associated gene expressions.

\section{Results}

\subsection{Morphological Features and $\beta$-Glucan Levels for Different Sized Beech Mushrooms}

The morphological aspects and different sizes (small, medium, and large) of the beech mushroom strains analyzed in this study are displayed in Figure 1A. The diameter of the caps and length of the stipes from the beech mushroom samples were measured, and they exhibited a linearly increasing trend with size (Table S1). The content of $\beta$-glucans in the cap were higher than those in the stipe samples, and these levels were proportionally increased with size with not significantly different. However, the $\beta$-glucan contents of the stipe decreased after the initial observed increase from small to medium sizes with no significance (Figure 1B).

(A)

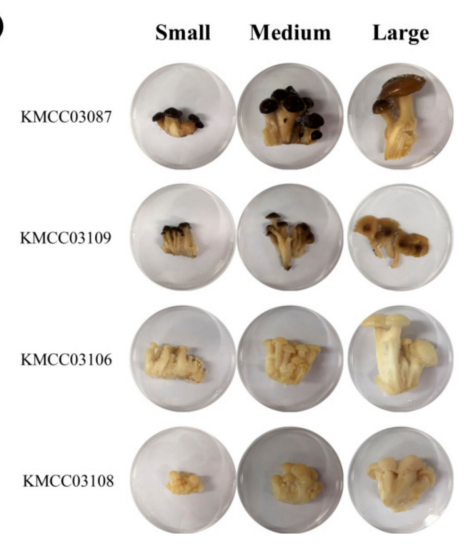

(B)

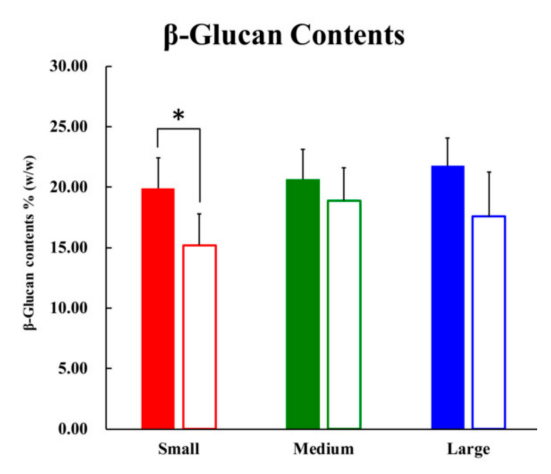

Figure 1. (A) Photographs of four beech mushrooms of different sizes. Developmental processes were divided according to three sizes: Small size; medium size; large size. (B) The graph represents the average $\beta$-glucan content. Data are represented as means \pm S.D, with three biological replicates $(n=3)$ maintained for each of the four mushroom strains used in the study. Significant differences between the cap and stipe were identified by $t$-test ( $p$-value $<0.05)$. $\mathbf{m}$, cap parts; $\square$, stipe parts. 


\subsection{Comprehensive Metabolite Profiles and Related Differentially Expressed Gene}

\subsubsection{Primary and Secondary Metabolites}

The GC-TOF-MS derived primary metabolite datasets for the caps and stipes of different sized (small, medium, and large) beech mushrooms were subjected to multivariate analyses incorporating the unsupervised PCA and the supervised PLS-DA. As shown in Figure 2A,C, the datasets for cap and stipe of small-sized mushroom samples were clustered apart from medium and large sized samples across PLS1 (15.92\% and 20.50\%), in respective score plots. On the other hand, both parts (cap and stipe) of medium-sized mushroom samples were segregated from small and large sizes along PLS2 (7.15\% and $5.25 \%$ ), in respective plots. We observed a similar consistency for the data patterns in PCA score plots (Figure S1A,C). Significantly different primary metabolites were identified and selected using variable importance in the projection value (VIP $>0.7)$ from PLS-DA models and $p$-value $(p<0.05)$ from one-way ANOVA. A total of 43 primary metabolites including 20 amino acids, one fatty acid, six organic acids, seven carbohydrates, four nucleotides, and five miscellaneous metabolites were characterized based on their retention times and mass fragment patterns data retrieved from National Institute of Standards and Technology (NIST, version 2.0., Gaithersburg, MD), Human metabolome Database (HMDB; http://www.hmdb.ca/), Wiley 9 and reference standards (Tables 1 and 2). We scored a level 2 confidence for the identified primary metabolites and a level 3 confidence for non-identified metabolites identified in the present study based on the annotation criteria suggested by the Chemical Analysis Working Group of the Metabolomics Standards Initiative [15]. The relative levels of primary metabolites among different sized beech mushrooms in both parts (cap and stipe) were expressed in a heat map scale and translated into fold-change values (Table 1). Considering the metabolite profiles for the cap portions, the relative abundance of 16 amino acids (except for glutamic acid, ornithine, and cystathionine), 2 nucleobases, and N.I. 1 were significantly higher in the small-sized beech mushrooms than those from medium- or large-sized samples. Conversely, three organic acids, four carbohydrates, adenosine-diphosphate (ADP), and four miscellaneous metabolites (benzoic acid, ethanolamine, urea, and acetyl-glucosamine) were significantly higher in the cap portions of the medium- and large-sized beech mushroom samples. Intriguingly, the pattern of relative abundance of significantly discriminant metabolites for the stipe portion of the mushrooms of different sizes was similar to that of the cap portion. Notably, the relative abundance of 16 amino acids (with the exception of ornithine and cystathionine), three nucleobases, glutaric acid, citric acid, acetyl-glucosamine, and N.I. One was higher in the small-sized mushroom stipes. On the other hand, three organic acids, five carbohydrates, one lipid, and benzoic acid were abundant in the stipe portions of the medium- and large-sized mushrooms.

The UHPLC-LTQ-IT-MS/MS datasets from negative ion mode were utilized to examine the disparity in secondary metabolites profiles of cap and stipe portions from different sized beech mushrooms. The orthogonal partial least squares discriminant analysis (OPLS-DA) score plots significantly indicated clear grouping patterns in both parts (cap and stipe) among the different sized mushrooms (Figure 2B,D). Based on OPLS-DA score plots, the datasets for both parts (cap and stipe) of small-sized mushroom samples were clearly segregated from medium and large sized samples across OPLS 1 (6.67\% and 8.84\%) in respective plots. Further, the datasets for medium sized samples were clustered separately from small and large sized samples across OPLS $2(3.08 \%$ and $3.74 \%$ ) in corresponding score plots. Unlike the primary metabolites profile based on GC-TOF-MS datasets, the PCA score plots for secondary metabolites did not indicate any differences among the mushroom samples of different sizes (Figure S1B,D). A total of nine secondary metabolites, including two dicarboxylic acids, 3 hypsiziprenols and four non-identified (N.I.) ones were selected as the significantly discriminant metabolites in cap and stipe portions among the different sized beech mushrooms at VIP $>0.7$ and $p$-value $<0.05$ based on OPLS-DA models (Table 1 ). These secondary metabolites were putatively identified based on retention time, MS/MS fragment pattern, standard compounds, and published studies with level 2 confidence of annotation, while the non-identified 
metabolites scored by level 3 [15]. The relative levels of discriminant metabolites were displayed in a heat map scale (Tables 1 and 2).
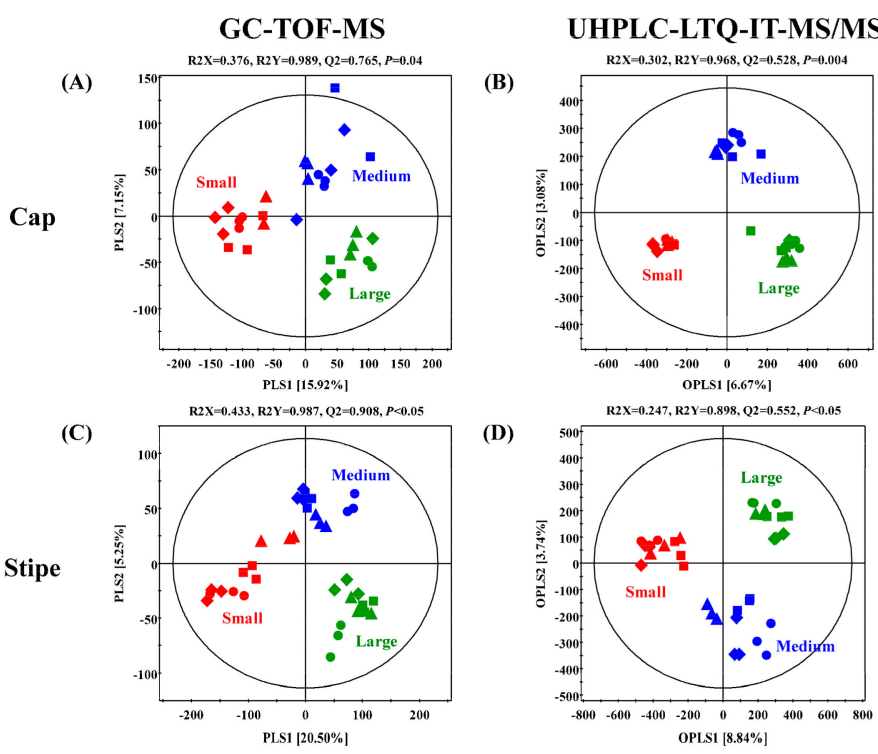

Figure 2. Partial least squares discriminant analysis (PLS-DA) and orthogonal partial least-discriminant analysis (OPLS-DA) score plots of caps $(\mathbf{A}, \mathbf{B})$ and stipes $(\mathbf{C}, \mathbf{D})$ collected, depending on different sizes of brown and white beech mushrooms, analyzed by GC-TOF-MS (A,C) and UHPLC-LTQ-IT-MS/MS (B and D). The biological replicates $(n=3)$ of four strain were analyzed by GC-TOF-MS and UHPLC-LTQ-IT-MS/MS. Small size is indicated by red color, medium size is indicated by blue

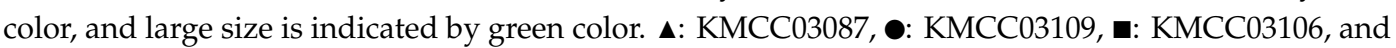
: KMCC03108.

Table 1. List of metabolites identified in caps and stipes of beech mushrooms of three different sizes.

\begin{tabular}{|c|c|c|c|c|c|c|c|}
\hline \multicolumn{8}{|c|}{ GC-TOF-MS (Primary Metabolites) } \\
\hline \multirow{2}{*}{ No. ${ }^{a}$} & \multirow{2}{*}{ Metabolite $^{\mathbf{b}}$} & \multicolumn{3}{|c|}{ Cap } & \multicolumn{3}{|c|}{ Stipe } \\
\hline & & Small & Medium & Large & Small & Medium & Large \\
\hline \multicolumn{8}{|c|}{ Amino acids } \\
\hline 1 & Alanine ** & 1.03 & 0.99 & 0.98 & 1.06 & 0.97 & 0.97 \\
\hline 2 & Valine & 1.21 & 0.87 & 0.91 & 1.19 & 0.94 & 0.87 \\
\hline 3 & Leucine ${ }^{* *}$ & 1.07 & 0.95 & 0.98 & 1.09 & 0.98 & 0.93 \\
\hline 4 & Isoleucine & 1.06 & 0.95 & 0.99 & 1.07 & 0.99 & 0.95 \\
\hline 5 & Proline & 1.09 & 0.95 & 0.96 & 1.06 & 1.00 & 0.94 \\
\hline 6 & Glycine & 1.08 & 0.94 & 0.97 & 1.04 & 1.01 & 0.95 \\
\hline 7 & Serine & 1.23 & 0.86 & 0.90 & 1.22 & 0.95 & 0.83 \\
\hline 8 & Threonine & 1.14 & 0.91 & 0.95 & 1.10 & 0.99 & 0.91 \\
\hline 9 & Methionine & 1.26 & 0.86 & 0.88 & 1.27 & 0.99 & 0.74 \\
\hline 10 & Pyroglutamic acid $* *$ & 1.04 & 1.00 & 0.96 & 1.07 & 0.98 & 0.95 \\
\hline 11 & Cysteine & 1.31 & 0.73 & 0.96 & 1.42 & 0.95 & 0.64 \\
\hline 12 & Glutamine * & 1.47 & 0.82 & 0.71 & 1.30 & 0.88 & 0.82 \\
\hline 13 & Glutamine acid * & 0.96 & 1.04 & 0.99 & 0.95 & 1.03 & 1.03 \\
\hline 14 & Phenylalanine & 1.04 & 0.98 & 0.99 & 1.06 & 1.00 & 0.94 \\
\hline 15 & Asparagine & 1.29 & 0.93 & 0.78 & 1.24 & 0.98 & 0.78 \\
\hline 16 & Ornithine & 0.60 & 1.16 & 1.24 & 0.73 & 1.08 & 1.19 \\
\hline 17 & Histidine * & 1.18 & 0.88 & 0.94 & 1.04 & 0.96 & 1.00 \\
\hline 18 & Tyrosine & 1.10 & 0.88 & 1.01 & 1.16 & 0.96 & 0.88 \\
\hline 19 & Cystathionine & 0.59 & 1.20 & 1.21 & 0.69 & 1.04 & 1.27 \\
\hline 20 & Tryptophan * & 1.17 & 0.86 & 0.97 & 1.11 & 0.99 & 0.90 \\
\hline
\end{tabular}


Table 1. Cont.

\begin{tabular}{|c|c|c|c|c|c|c|c|}
\hline \multicolumn{8}{|c|}{ Fatty acids } \\
\hline 21 & Oleamide ${ }^{* *}$ & 0.91 & 0.91 & 1.18 & 0.82 & 0.79 & 1.39 \\
\hline \multicolumn{8}{|c|}{ Organic acids } \\
\hline 22 & Lactic acid ** & 0.79 & 0.99 & 1.22 & 0.77 & 0.89 & 1.34 \\
\hline 23 & Succinic acid * & 0.73 & 1.02 & 1.25 & 1.08 & 1.03 & 0.89 \\
\hline 24 & Fumaric acid & 0.71 & 1.17 & 1.12 & 0.83 & 1.04 & 1.13 \\
\hline 25 & Malic acid & 0.75 & 1.11 & 1.14 & 0.85 & 1.05 & 1.10 \\
\hline 26 & Glutaric acid ** & 1.12 & 1.07 & 0.81 & 1.84 & 0.68 & 0.48 \\
\hline 27 & Citric acid ${ }^{* *}$ & 0.90 & 1.14 & 0.96 & 1.47 & 0.60 & 0.93 \\
\hline \multicolumn{8}{|c|}{ Carbohydrates } \\
\hline 28 & Glycerol * & 1.00 & 0.88 & 1.12 & 1.03 & 0.98 & 1.00 \\
\hline 29 & Glyceric acid * & 0.82 & 0.95 & 1.24 & 1.02 & 0.94 & 1.03 \\
\hline 30 & Fructose ${ }^{* *}$ & 0.82 & 0.89 & 1.29 & 0.73 & 0.91 & 1.36 \\
\hline 31 & Glucose ${ }^{* *}$ & 0.82 & 0.93 & 1.24 & 0.79 & 1.01 & 1.21 \\
\hline 32 & Gluconic acid ** & 0.90 & 1.16 & 0.94 & 0.95 & 1.22 & 0.83 \\
\hline 33 & myo-Inositiol & 0.93 & 1.04 & 1.03 & 0.93 & 1.00 & 1.06 \\
\hline 34 & Glucose 6-phosphate & 0.70 & 1.12 & 1.19 & 0.73 & 1.25 & 1.02 \\
\hline \multicolumn{8}{|c|}{ Nucleosides } \\
\hline 35 & Adenosine-diphosphate & 0.92 & 1.05 & 1.03 & 0.93 & 1.00 & 1.06 \\
\hline 36 & Uridine $^{* *}$ & 1.16 & 0.96 & 0.88 & 1.38 & 0.89 & 0.73 \\
\hline 37 & Guanine & 1.18 & 0.93 & 0.90 & 1.24 & 0.94 & 0.82 \\
\hline 38 & Adenosine & 1.18 & 0.92 & 0.90 & 1.27 & 0.93 & 0.80 \\
\hline \multicolumn{8}{|c|}{ Miscellaneous } \\
\hline 39 & Benzoic acid & 0.92 & 1.03 & 1.05 & 0.96 & 0.97 & 1.06 \\
\hline 40 & Ethanolamine * & 0.87 & 0.95 & 1.18 & 1.07 & 0.91 & 1.02 \\
\hline 41 & Urea * & 0.82 & 1.03 & 1.15 & 1.12 & 0.94 & 0.94 \\
\hline 42 & Acetyl-glucosamine & 1.01 & 0.85 & 1.14 & 1.29 & 0.97 & 0.74 \\
\hline 43 & N.I 1 & 1.15 & 1.08 & 0.77 & 1.35 & 0.94 & 0.71 \\
\hline \multicolumn{8}{|c|}{ UHPLC-LTQ-IT-MS/MS (Secondary metabolites) } \\
\hline \multirow{2}{*}{ No. ${ }^{a}$} & \multirow{2}{*}{ Metabolite $^{\mathrm{c}}$} & \multicolumn{3}{|c|}{ Cap } & \multicolumn{3}{|c|}{ Stipe } \\
\hline & & Small & Medium & Large & Small & Medium & Large \\
\hline \multicolumn{8}{|c|}{ Saturated dicarboxylic acids } \\
\hline 44 & Azelaic acid * & 1.07 & 0.87 & 1.06 & 1.67 & 0.68 & 0.65 \\
\hline 45 & Sebacic acid ${ }^{* *}$ & 1.07 & 0.96 & 0.98 & 1.22 & 0.96 & 0.82 \\
\hline \multicolumn{8}{|c|}{ Hypsiziprenols } \\
\hline 46 & Hypsiziprenol A14 * & 0.90 & 0.92 & 1.18 & 1.13 & 1.00 & 0.87 \\
\hline 47 & Hypsiziprenol A13 ** & 0.99 & 0.92 & 1.09 & 1.21 & 0.99 & 0.80 \\
\hline 48 & Hypsiziprenol A12 ** & 0.99 & 0.97 & 1.04 & 1.19 & 1.04 & 0.77 \\
\hline \multicolumn{8}{|c|}{ Non-identifications } \\
\hline 49 & N.I. $2 * *$ & 1.02 & 1.07 & 0.91 & 1.22 & 1.19 & 0.59 \\
\hline 50 & N.I. 3 & 0.62 & 0.87 & 1.51 & 0.56 & 0.67 & 1.77 \\
\hline 51 & N.I. 4 * & 0.82 & 0.95 & 1.23 & 1.03 & 1.07 & 0.90 \\
\hline 52 & N.I. $5^{* *}$ & 1.04 & 0.94 & 1.02 & 1.19 & 0.97 & 0.84 \\
\hline
\end{tabular}

${ }^{\mathrm{a}}$ Number of metabolites.; ${ }^{\mathrm{b}}$ Selected and identified primary metabolites based on variable importance in projection $(\mathrm{VIP})$ value $(>0.7)$ and $p$-value $(<0.05)$ in both VIP1 and VIP2 by PLS-DA.; ${ }^{\mathrm{c}}$ Selected and identified secondary metabolites based on variable importance in projection (VIP) value $(>0.7)$ and $p$-value $(<0.05)$ in both VIP1 and VIP2 by OPLS-DA.; Only selected metabolites in caps.; ${ }^{* *}$ Only selected metabolites in stipes.; The colored squares (blue-to-red) represent fold changes normalized by average of each metabolites of beech mushroom. The color scheme is as follows: Lower limit value, 0 (blue); middle limit value, 1 (white); upper limit value, 1.5 (red). 
Table 2. Tentative identified primary and secondary metabolites in caps and stipes of beech mushrooms of three different sizes based on GC-TOF-MS and UHPLC-LTQ-IT-MS/MS.

\begin{tabular}{|c|c|c|c|c|c|c|c|c|c|c|c|c|c|}
\hline \multicolumn{6}{|c|}{ GC-TOF-MS } & \multicolumn{8}{|c|}{ UHPLC-LTQ-IT-MS/MS } \\
\hline No. ${ }^{a}$ & Metabolite $^{\mathrm{b}}$ & $\mathbf{R t}^{\mathrm{c}}{ }_{(\min )}$ & $\begin{array}{c}\text { Mass Fragment } \\
\text { Pattern }(m / z)\end{array}$ & TMS $^{d}$ & ID & No. ${ }^{a}$ & Metabolite $^{\mathrm{e}}$ & $\begin{array}{l}\mathbf{R t}^{\mathrm{c}} \\
(\min )\end{array}$ & {$[\mathrm{M}-\mathrm{H}]^{-}$} & {$[\mathrm{M}+\mathrm{H}]^{+}$} & M.W. & $\begin{array}{c}\text { MS }^{\mathrm{n}}[\mathrm{M}-\mathrm{H}]- \\
\text { Fragment Pattern }\end{array}$ & ID \\
\hline \multicolumn{6}{|c|}{ Amino acids } & \multicolumn{8}{|c|}{ Saturated dicarboxylic acids } \\
\hline 1 & Alanine ** & 5.15 & $100,103,116,190$ & $(\mathrm{TMS})_{2}$ & STD/MS & 44 & Azelaic acid* & 8.36 & 187 & - & 188 & $187>125>97$ & STD \\
\hline 2 & Valine & 6.64 & $45,59,100,144,218$ & $(\mathrm{TMS})_{2}$ & STD/MS & 45 & Sebacic acid ** & 9.40 & 201 & - & 202 & $201>183,139$ & STD \\
\hline 3 & Leucine ${ }^{* *}$ & 7.20 & $73,100,102,133,158$ & $(\mathrm{TMS})_{2}$ & STD/MS & \multicolumn{8}{|c|}{ Hypsiziprenols } \\
\hline 4 & Isoleucine & 7.42 & $59,73,100,158,218$ & $(\mathrm{TMS})_{2}$ & STD/MS & 46 & Hypsiziprenol A14* & 14.42 & 1214 & 1170 & 1169 & $1168>1098>1080,1014$ & REF \\
\hline 5 & Proline & 7.47 & $59,73,142,147$ & $(\mathrm{TMS})_{2}$ & STD/MS & 47 & Hypsiziprenol A13 ** & 14.48 & 1082 & 1084 & 1083 & $1082>300>882,816$ & REF \\
\hline 6 & Glycine & 7.55 & $86,100,133,174,248$ & $(\mathrm{TMS})_{3}$ & STD/MS & 48 & Hypsiziprenol A12 ** & 14.56 & 996 & 998 & 997 & $996>814>956,730$ & REF \\
\hline 7 & Serine & 8.05 & $73,100,147,204,218$ & $(\mathrm{TMS})_{3}$ & STD/MS & \multicolumn{8}{|c|}{ Non-identifications } \\
\hline 8 & Threonine & 8.31 & $73,101,117,129,219$ & $(\mathrm{TMS})_{3}$ & STD/MS & 49 & N.I. 2 ** & 6.22 & 492 & 494 & 493 & $494>474>254$ & - \\
\hline 9 & Methionine & 9.45 & $61,100,128,147,176$ & $(\mathrm{TMS})_{2}$ & STD/MS & 50 & N.I. 3 & 8.16 & 432 & 434 & 433 & $434>306>288,272,254$ & - \\
\hline 10 & Pyroglutamic acid $* *$ & 9.51 & $59,147,156,230,258$ & $(\mathrm{TMS})_{2}$ & STD/MS & 51 & N.I. $4 *$ & 9.05 & 199 & 201 & 200 & - & - \\
\hline 11 & Cysteine & 9.75 & $73,100,147,218,220$ & $(\mathrm{TMS})_{3}$ & STD/MS & 52 & N.I. $5^{* *}$ & 10.71 & 329 & - & - & - & - \\
\hline 12 & Glutamine * & 10.07 & $128,139,147,154,227$ & $(\mathrm{TMS})_{3}$ & STD/MS & & & & & & & & \\
\hline 13 & Glutamine acid * & 10.24 & $56,84,127,156,246$ & $(\mathrm{TMS})_{3}$ & STD/MS & & & & & & & & \\
\hline 14 & Phenylalanine & 10.34 & $73,100,147,192,218$ & $(\mathrm{TMS})_{2}$ & STD/MS & & & & & & & & \\
\hline 15 & Asparagine & 10.66 & $73,116,132,141,188$ & $(\mathrm{TMS})_{3}$ & STD/MS & & & & & & & & \\
\hline 16 & Ornithine & 11.73 & $73,142,147,174,100$ & $(\mathrm{TMS})_{4}$ & STD/MS & & & & & & & & \\
\hline 17 & Histidine* & 12.48 & $74,100,107,154,254$ & $(\mathrm{TMS})_{3}$ & STD/MS & & & & & & & & \\
\hline 18 & Tyrosine & 12.57 & $73,100,147,218$ & $(\mathrm{TMS})_{3}$ & STD/MS & & & & & & & & \\
\hline 19 & Cystathionine & 14.26 & $73,100,128,147,218$ & $(\mathrm{TMS})_{4}$ & STD/MS & & & & & & & & \\
\hline 20 & Tryptophan * & 14.36 & $73,100,147,202,218$ & $(\mathrm{TMS})_{3}$ & STD/MS & & & & & & & & \\
\hline \multicolumn{6}{|c|}{ Fatty acids } & & & & & & & & \\
\hline 21 & Oleamide ** & 15.33 & $116,128,131,144,198$ & $(\mathrm{TMS})_{1}$ & STD/MS & & & & & & & & \\
\hline \multicolumn{6}{|c|}{ Organic acids } & & & & & & & & \\
\hline 22 & Lactic acid $* *$ & 4.99 & $59,75,117,147,191$ & $(\mathrm{TMS})_{2}$ & STD/MS & & & & & & & & \\
\hline 23 & Succinic acid * & 7.58 & $55,73,129,147,247$ & $(\mathrm{TMS})_{2}$ & STD/MS & & & & & & & & \\
\hline 24 & Fumaric acid & 7.83 & $75,115,143,147,245$ & $(\mathrm{TMS})_{2}$ & STD/MS & & & & & & & & \\
\hline 25 & Malic acid & 9.19 & $55,101,133,147,233$ & $(\mathrm{TMS})_{3}$ & STD/MS & & & & & & & & \\
\hline 26 & Glutaric acid ** & 9.89 & $45,55,112,156,198$ & $(\mathrm{TMS})_{2}$ & STD/MS & & & & & & & & \\
\hline 27 & Citric acid ** & 11.76 & $45,67,73,147,273$ & $(\mathrm{TMS})_{4}$ & STD/MS & & & & & & & & \\
\hline
\end{tabular}


Table 2. Cont

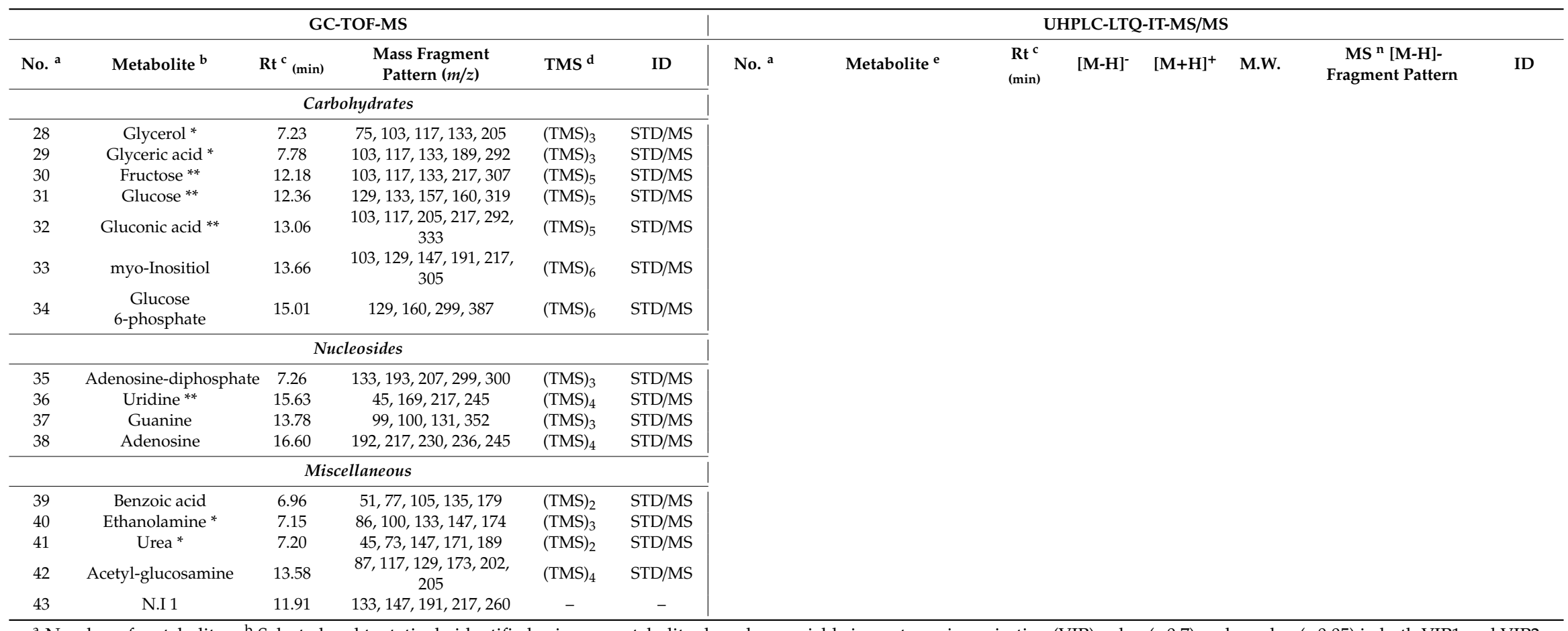

${ }^{a}$ Number of metabolites.; ${ }^{b}$ Selected and tentatively identified primary metabolites based on variable importance in projection (VIP) value ( $\left.>0.7\right)$ and $p$-value $(<0.05)$ in both VIP1 and VIP 2

by PLS-DA.; ${ }^{\mathrm{c}}$ Retention time.; ${ }^{\mathrm{d}}$ TMS, the number of trimethylsilyl groups; ${ }^{\mathrm{e}}$ Selected and tentatively identified secondary metabolites based on variable importance in projection (VIP) value $(>0.7)$ and $p$-value $(<0.05)$ in both VIP1 and VIP2 by OPLS-DA.; ${ }^{\mathrm{f}}$ Identification. STD/MS, comparing with standard compounds analyzed under same condition and mass spectra comparison with HMDB, NIST database, and Wiley 9.; $\mathrm{g}$ Identification. STD, comparing with standard compounds analyzed under same condition; REF, published paper ([3] Park et al., 2017); CCD, Dictionary of Natural product; ${ }^{\mathrm{h}}[\mathrm{H}+\mathrm{HCOOH}-\mathrm{H}]^{-} ;{ }^{*}$ Only selected metabolites in caps (VIP $\left.>0.7, p<0.05\right)$.; ${ }^{* *}$ Only selected metabolites in stipes (VIP $\left.>0.7, p<0.05\right)$. 


\subsubsection{Significantly Ciscriminant Metabolites-Related Differentially Expressed Genes}

One-way ANOVA ( $p$-value $<0.05$ ) for RNA-sequencing data indicated 38 differentially expressed genes (DEGs) representing eight different metabolic pathways in different sized mushroom samples. Categorically, we observed different number of DEGs linked to the metabolism of carbohydrates (11), amino acids (7), nucleotides (4), terpenoids (4), lipids (3), xenobiotics biodegradation (3), other amino acids (2), and some miscellaneous metabolites (4). The DEGs representing various metabolic pathways for beech mushroom samples are listed in Table 3 with corresponding gene IDs, annotations, and enzyme commission (EC) numbers retrieved from the Kyoto Encyclopedia of Genes and Genomes (KEGG) pathway database.

The heat map data (Table 3) displays the linear increase in the expression levels of DEGs linked with the carbohydrate metabolism in beech mushroom according to size irrespective of the spatial components (cap and stipe) except for $\beta$-glucuronidase (EC 3.2.1.31), FAD-linked oxidoreductase (EC 1.1.3.15), laccase-1 (EC 1.10.3.2), and NADP-dependent malic enzyme (EC 1.1.1.40). Similarly, DEGs associated with xenobiotics biodegradation metabolism were highly expressed in medium and large sized mushrooms except for 3-hydroxy-phenylacetate 6-hydroxylase (EC 1.14.13.63). In contrast, seven amino acids, four nucleotides, four terpenoids, three lipids, and two other amino acid metabolism-related genes were lower expressed with increase in mushroom sizes notwithstanding their spatial variations. However, DEGs for meiotically up-regulated gene 158 protein (EC 2.1.1.44 and 1.14.99.51) displayed higher abundance in large sized (cap and stipe) mushrooms, while those for lipase 4 (EC 3.1.1.3) were abundant in cap (large sized) and stipe (small sized) mushrooms. Further, the relative abundance of DEGs associated with carbonic anhydrase (EC 4.2.1.1) in nitrogen metabolism and 4-coumarate CoA ligase (EC 6.2.1.12) in phenylpropanoid biosynthesis were relatively higher expressed in large sized mushrooms; whereas, phenylalanine-tRNA ligase (EC 6.1.1.20) in aminoacyl-tRNA biosynthesis and ferric reductase transmembrane component 5 (EC 1.16.1.7) in ferric chelate reduction were higher expressed in small-sized beech mushrooms. 
Table 3. Differentially expressed genes (DEGs) related to metabolite biosyntheses in three different sized beech mushrooms.

\begin{tabular}{|c|c|c|c|c|c|c|c|c|c|c|}
\hline \multirow{2}{*}{ No. } & \multirow{2}{*}{ Gene ID $^{a}$} & \multirow{2}{*}{ Sub-Classification } & \multirow{2}{*}{ Annotation ${ }^{b}$} & \multirow{2}{*}{ EC number ${ }^{c}$} & \multicolumn{3}{|c|}{ Cap } & \multicolumn{3}{|c|}{ Stipe } \\
\hline & & & & & Small & Medium & Large & Small & Medium & Large \\
\hline \multicolumn{11}{|c|}{ Carbohydrate Metabolism } \\
\hline 1 & Htessellatus1SL000147t0011 & $\begin{array}{c}\text { Ascorbate and } \\
\text { aldarate metabolism }\end{array}$ & $\begin{array}{c}\text { Versatile peroxidase } \\
\text { VPL1 }\end{array}$ & 1.11.1.13 & 0.78 & 0.91 & 1.30 & 0.76 & 0.91 & 1.33 \\
\hline 2 & Htessellatus1SL000266t0019 & $\begin{array}{l}\text { Butanoate } \\
\text { metabolism }\end{array}$ & $\begin{array}{c}\text { L-2-Hydroxyglutarate } \\
\text { dehydrogenase }\end{array}$ & 1.1.99.2 & 0.67 & 0.91 & 1.43 & 0.63 & 0.96 & 1.41 \\
\hline 3 & Htessellatus1SL008026t0008 & $\begin{array}{l}\text { Glyoxylate and } \\
\text { dicarboxylate } \\
\text { metabolism }\end{array}$ & Malate synthase & 2.3.3.9 & 0.61 & 0.77 & 1.61 & 0.50 & 0.96 & 1.54 \\
\hline 4 & Htessellatus1SL013065t0001 & $\begin{array}{l}\text { Starch and sucrose } \\
\text { metabolism }\end{array}$ & $\begin{array}{c}\text { Putative } \beta \text {-glucan } \\
\text { synthesis-associated } \\
\text { protein }\end{array}$ & KPE6 & 0.69 & 0.87 & 1.45 & 0.63 & 0.87 & 1.50 \\
\hline 5 & Htessellatus1SL006041t0015 & $\begin{array}{l}\text { Starch and sucrose } \\
\text { metabolism }\end{array}$ & 1,3 - $\beta$-glucan synthase & 2.4.1.34 & 0.69 & 0.95 & 1.36 & 0.69 & 1.03 & 1.28 \\
\hline 6 & Htessellatus1SL008799t0004 & $\begin{array}{c}\text { Fructose and } \\
\text { mannose metabolism }\end{array}$ & $\begin{array}{c}\text { NADP-dependent } \\
\text { mannitol } \\
\text { dehydrogenase }\end{array}$ & 1.1.1.138 & 0.49 & 0.70 & 1.81 & 0.49 & 0.64 & 1.86 \\
\hline 7 & Htessellatus1SL005645t0003 & $\begin{array}{c}\text { Pentose and } \\
\text { glucuronate } \\
\text { interconversions }\end{array}$ & $\begin{array}{c}\text { Putative } \\
\text { NAD }(\mathrm{P}) \mathrm{H} \text {-dependent } \\
\text { D-xylose reductase }\end{array}$ & 1.1.1.307 & 0.77 & 0.83 & 1.40 & 0.47 & 0.89 & 1.65 \\
\hline 8 & Htessellatus1SL007796t0013 & $\begin{array}{c}\text { Pentose and } \\
\text { glucuronate } \\
\text { interconversions }\end{array}$ & $\beta$-Glucuronidase & 3.2.1.31 & 1.48 & 0.94 & 0.58 & 1.09 & 0.90 & 1.02 \\
\hline 9 & Htessellatus1SL004183t0003 & $\begin{array}{c}\text { Ascorbate and } \\
\text { aldarate metabolism }\end{array}$ & Laccase-1 & 1.10.3.2 & 1.26 & 1.03 & 0.71 & 1.15 & 0.96 & 0.89 \\
\hline 10 & Htessellatus1SL000110t0005 & $\begin{array}{l}\text { Glyoxylate and } \\
\text { dicarboxylate } \\
\text { metabolism }\end{array}$ & $\begin{array}{c}\text { FAD-linked } \\
\text { oxidoreductase }\end{array}$ & 1.1.3.15 & 1.72 & 0.90 & 0.37 & 1.21 & 1.11 & 0.68 \\
\hline 11 & Htessellatus1SL004210t0003 & Pyruvate metabolism & $\begin{array}{l}\text { NADP-dependent } \\
\text { malic enzyme }\end{array}$ & 1.1.1.40 & 1.15 & 1.04 & 0.81 & 1.52 & 0.99 & 0.49 \\
\hline
\end{tabular}


Table 3. Cont

\begin{tabular}{|c|c|c|c|c|c|c|c|c|c|c|}
\hline \multirow{2}{*}{ No. } & \multirow{2}{*}{ Gene ID ${ }^{a}$} & \multirow{2}{*}{ Sub-Classification } & \multirow{2}{*}{ Annotation $b$} & \multirow{2}{*}{ EC number ${ }^{c}$} & \multicolumn{3}{|c|}{ Cap } & \multicolumn{3}{|c|}{ Stipe } \\
\hline & & & & & Small & Medium & Large & Small & Medium & Large \\
\hline \multicolumn{11}{|c|}{ Amino acid metabolism } \\
\hline 12 & Htessellatus1SL006626t0002 & Histidine metabolism & $\begin{array}{c}\text { Meiotically } \\
\text { up-regulated gene } 158 \\
\text { protein }\end{array}$ & $\begin{array}{r}2.1 .1 .44 / \\
1.14 .99 .51\end{array}$ & 0.41 & 0.78 & 1.80 & 0.43 & 0.96 & 1.61 \\
\hline 13 & Htessellatus1SL007811t0003 & Histidine metabolism & $\begin{array}{l}\text { Putative bifunctional } \\
\text { amine oxidase }\end{array}$ & 1.4.3.4 & 1.33 & 1.27 & 0.40 & 1.17 & 0.92 & 0.91 \\
\hline 14 & Htessellatus1SL001236t0007 & $\begin{array}{l}\text { Cysteine and } \\
\text { methionine } \\
\text { metabolism }\end{array}$ & $\begin{array}{l}\text { Putative cystathionine } \\
\text { gamma-synthase }\end{array}$ & 2.5.1.48 & 1.21 & 0.94 & 0.85 & 1.49 & 0.97 & 0.54 \\
\hline 15 & Htessellatus1SL005032t0006 & $\begin{array}{l}\text { Glycine, serine and } \\
\text { threonine metabolism }\end{array}$ & $\begin{array}{l}\text { D-3-phosphoglycerate } \\
\text { dehydrogenase } 1\end{array}$ & 1.1.1.95 & 1.23 & 0.87 & 0.90 & 1.24 & 0.98 & 0.78 \\
\hline 16 & Htessellatus1SL002183t0001 & $\begin{array}{l}\text { Phenylalanine, } \\
\text { tyrosine and } \\
\text { tryptophan } \\
\text { biosynthesis }\end{array}$ & Tryptophan synthase & 4.2.1.20 & 1.23 & 1.03 & 0.74 & 1.13 & 1.13 & 0.74 \\
\hline 17 & Htessellatus1SL009909t0005 & Tyrosine metabolism & Tyrosinase & 1.14.18.1 & 1.77 & 0.88 & 0.35 & 1.37 & 1.01 & 0.62 \\
\hline 18 & Htessellatus1SL006526t0001 & $\begin{array}{l}\text { Valine, leucine and } \\
\text { isoleucine } \\
\text { biosynthesis }\end{array}$ & $\begin{array}{l}\text { Putative ketol acid } \\
\text { reductoisomerase }\end{array}$ & 1.1.1.86 & 1.22 & 0.96 & 0.83 & 1.13 & 1.04 & 0.82 \\
\hline \multicolumn{11}{|c|}{ Nucleotide metabolism } \\
\hline 19 & Htessellatus1SL014146t0001 & $\begin{array}{l}\text { Pyrimidine } \\
\text { metabolism }\end{array}$ & $\begin{array}{l}\text { Ribonucleoside-diphos } \\
\text { reductase small chain }\end{array}$ & nate 1.17 .4 .1 & 1.21 & 1.00 & 0.79 & 1.45 & 0.98 & 0.57 \\
\hline 20 & Htessellatus1SL002783t0004 & $\begin{array}{l}\text { Pyrimidine \& Purine } \\
\text { metabolism }\end{array}$ & $\begin{array}{l}\text { Ribonucleoside } \\
\text { diphosphate } \\
\text { reductase large chain }\end{array}$ & 1.17.4.1 & 1.19 & 1.02 & 0.79 & 1.36 & 1.04 & 0.60 \\
\hline 21 & Htessellatus1SL006389t0001 & $\begin{array}{c}\text { Pyrimidine \& Purine } \\
\text { metabolism }\end{array}$ & Golgi apyrase & 3.6.1.5 & 1.57 & 1.02 & 0.42 & 1.40 & 1.10 & 0.50 \\
\hline 22 & Htessellatus1SL012650t0011 & Purine metabolism & $\begin{array}{c}\text { Putative } \\
\text { exopolyphosphatase }\end{array}$ & 3.6.1.11 & 1.24 & 1.01 & 0.75 & 1.44 & 1.03 & 0.53 \\
\hline
\end{tabular}


Table 3. Cont

\begin{tabular}{|c|c|c|c|c|c|c|c|c|c|c|}
\hline \multirow{2}{*}{ No. } & \multirow{2}{*}{ Gene ID ${ }^{a}$} & \multirow{2}{*}{ Sub-Classification } & \multirow{2}{*}{ Annotation ${ }^{b}$} & \multirow{2}{*}{ EC number ${ }^{c}$} & \multicolumn{3}{|c|}{ Cap } & \multicolumn{3}{|c|}{ Stipe } \\
\hline & & & & & Small & Medium & Large & Small & Medium & Large \\
\hline \multicolumn{11}{|c|}{ Terpenoid metabolism } \\
\hline 26 & Htessellatus1SL002734t0005 & $\begin{array}{l}\text { Monoterpenoid } \\
\text { biosynthesis }\end{array}$ & $\begin{array}{l}\text { Neomenthol } \\
\text { dehydrogenase }\end{array}$ & 1.1.1.208 & 1.25 & 0.98 & 0.77 & 1.37 & 1.06 & 0.57 \\
\hline 23 & Htessellatus1SL007764t0004 & $\begin{array}{c}\text { Terpenoid backbone } \\
\text { biosynthesis }\end{array}$ & $\begin{array}{l}\text { Farnesyl } \\
\text { pyrophosphate } \\
\text { synthase }\end{array}$ & $\begin{array}{l}2.5 .1 .1 / \\
2.5 .1 .10\end{array}$ & 1.23 & 0.97 & 0.8 & 1.12 & 1.04 & 0.84 \\
\hline 24 & Htessellatus1SL001218t0008 & $\begin{array}{l}\text { Terpenoid backbone } \\
\text { biosynthesis }\end{array}$ & $\begin{array}{c}\text { Geranylgeranyl } \\
\text { diphosphate synthase }\end{array}$ & 2.5.1.29 & 1.48 & 1.09 & 0.42 & 1.72 & 0.96 & 0.33 \\
\hline 25 & Htessellatus1SL000034t0003 & $\begin{array}{c}\text { Terpenoid backbone } \\
\text { biosynthesis }\end{array}$ & $\begin{array}{l}\text { Short chain isoprenyl } \\
\text { diphosphate synthase }\end{array}$ & $\begin{array}{c}2.5 .1 .1 / \\
2.5 .1 .10 / \\
2.5 .1 .29\end{array}$ & 1.34 & 1.01 & 0.64 & 1.27 & 1.06 & 0.67 \\
\hline \multicolumn{11}{|c|}{ Lipid metabolism } \\
\hline 27 & Htessellatus1SL005093t0001 & $\begin{array}{l}\text { Glycerolipid } \\
\text { metabolism }\end{array}$ & Lipase 4 & 3.1.1.3 & 0.33 & 0.94 & 1.73 & 1.43 & 1.21 & 0.35 \\
\hline 28 & Htessellatus1SL000281t0004 & Steroid biosynthesis & Lathosterol oxidase & 1.14.19.20 & 1.19 & 0.99 & 0.83 & 1.20 & 0.96 & 0.85 \\
\hline 29 & Htessellatus1SL000053t0001 & Steroid biosynthesis & $\begin{array}{l}\text { Methylsterol } \\
\text { monooxygenase }\end{array}$ & 1.14.18.9 & 1.20 & 1.02 & 0.77 & 1.16 & 1.08 & 0.77 \\
\hline \multicolumn{11}{|c|}{ Xenobiotics biodegradation and metabolism } \\
\hline 30 & Htessellatus1SL003043t0001 & $\begin{array}{c}\text { Aminobenzoate } \\
\text { degradation }\end{array}$ & $\begin{array}{l}\text { Benzoylformate } \\
\text { decarboxylase }\end{array}$ & 4.1.1.7 & 0.69 & 0.68 & 1.63 & 0.66 & 0.92 & 1.41 \\
\hline 31 & Htessellatus1SL010123t0002 & Benzoate degradation & $\begin{array}{c}\text { Benzoate } \\
\text { 4-monooxygenase }\end{array}$ & 1.14.13.12 & 0.67 & 0.91 & 1.42 & 0.54 & 0.90 & 1.57 \\
\hline 32 & Htessellatus1SL006522t0004 & Styrene degradation & $\begin{array}{l}\text { 3-Hydroxyphenylacetate } \\
\text { 6-hydroxylase }\end{array}$ & 1.14.13.63 & 1.32 & 1.10 & 0.57 & 1.81 & 0.84 & 0.35 \\
\hline
\end{tabular}


Table 3. Cont.

\begin{tabular}{|c|c|c|c|c|c|c|c|c|c|c|}
\hline \multirow{2}{*}{ No. } & \multirow{2}{*}{ Gene ID ${ }^{a}$} & \multirow{2}{*}{ Sub-Classification } & \multirow{2}{*}{ Annotation $^{b}$} & \multirow{2}{*}{ EC number ${ }^{c}$} & \multicolumn{3}{|c|}{ Cap } & \multicolumn{3}{|c|}{ Stipe } \\
\hline & & & & & Small & Medium & Large & Small & Medium & Large \\
\hline \multicolumn{11}{|c|}{ Other amino acid metabolism } \\
\hline 34 & Htessellatus1SL004301t0002 & $\begin{array}{l}\text { Glutathione } \\
\text { metabolism }\end{array}$ & Peroxiredoxin & 1.11.1.15 & 1.23 & 0.99 & 0.78 & 1.36 & 1.01 & 0.64 \\
\hline 33 & Htessellatus1SL000068t0001 & $\begin{array}{l}\text { Peptidylprolyl } \\
\text { isomerase }\end{array}$ & $\begin{array}{l}\text { FK506-binding } \\
\text { protein } 4\end{array}$ & 5.2.1.8 & 1.32 & 0.95 & 0.73 & 1.35 & 1.01 & 0.64 \\
\hline \multicolumn{11}{|c|}{ Miscellaneous metabolism } \\
\hline 36 & Htessellatus1SL009373t0001 & Nitrogen metabolism & Carbonic anhydrase & 4.2.1.1 & 0.75 & 0.80 & 1.45 & 0.56 & 0.83 & 1.61 \\
\hline 35 & Htessellatus1SL006510t0001 & $\begin{array}{l}\text { Phenylpropanoid } \\
\text { biosynthesis }\end{array}$ & $\begin{array}{l}\text { 4-Coumarate CoA } \\
\text { ligase-like } 7\end{array}$ & 6.2.1.12 & 0.86 & 0.88 & 1.26 & 0.72 & 0.95 & 1.33 \\
\hline 37 & Htessellatus1SL002357t0005 & $\begin{array}{c}\text { Aminoacyl-tRNA } \\
\text { biosynthesis }\end{array}$ & $\begin{array}{c}\text { Phenylalanine-tRNA } \\
\text { ligase }\end{array}$ & 6.1.1.20 & 1.28 & 1.01 & 0.71 & 1.36 & 1.04 & 0.60 \\
\hline 38 & Htessellatus1SL012836t0001 & $\begin{array}{l}\text { Ferric-chelate } \\
\text { reductase }\end{array}$ & $\begin{array}{l}\text { Ferric reductase } \\
\text { transmembrane } \\
\text { component } 5\end{array}$ & 1.16.1.7 & 1.38 & 1.13 & 0.49 & 1.25 & 1.14 & 0.61 \\
\hline
\end{tabular}

${ }^{\mathrm{a}}$ Gene number is approximately the same as Hypsizygus marmoreus, which suggests a similar matched gene number in mushroom family. Genes were selected by $p$-value $<0.05 .{ }^{\mathrm{b}}$ Biological information in sequences.; ${ }^{c}$ Enzyme commission number for enzyme from KEGG database (http://www.genome.jp/kegg/).; The colored squares (blue-to-red) represent fold changes normalized by the average of each gene expression of beech mushroom. The color scheme is as follows: Lower limit value, 0 (blue); middle limit value, 1 (white); upper limit value, 2 (red). 


\subsection{Integrated Pathway Mapping for Discriminant Metabolites and DEGs in Different Sized Beech Mushrooms}

The synergies in the relative abundance of metabolites and associated DEGs among the different sized beech mushroom samples were visualized in corresponding pathway maps (Figure 3). Notably, several metabolites and their corresponding DEGs exhibited metabolite-gene correlation depending on the different mushroom sizes. The relative abundance of metabolites and associated genes were well-correlated with each other, especially those involved in valine, leucine, and isoleucine biosyntheses mediated by ketol acid reductoisomerase (EC 1.1.1.86); cysteine and methionine biosyntheses mediated by cystathionine gamma-synthase (EC 2.5.1.48); tryptophan by tryptophan synthase (EC 4.2.1.20); tyrosine by tyrosinase (EC 1.14.18.1); glycine, serine, and threonine by D-3-phosphoglycerate dehydrogenase (EC 1.1.1.95); malic acid by malate synthase (EC 2.3.3.9), $\beta$-glucans by $\beta$-glucan synthesis-associated protein (KPE6) and 1, 3- $\beta$-glucan synthase (EC 2.4.1.13); nucleotides (uridine, guanine, and adenosine) by ribonucleoside-diphosphate reductases (EC 1.17.4.1), exopolyphosphatase (EC 3.6.1.11) and Golgi apyrase (EC 3.6.1.5); phenolic acids such as benzoic acid with 4-coumarate CoA ligase-like 7 (EC 6.2.1.12), benzoylformate decarboxylase (EC 4.1.1.7) and benzoate 4-monooxygenase (EC 1.14.13.12).

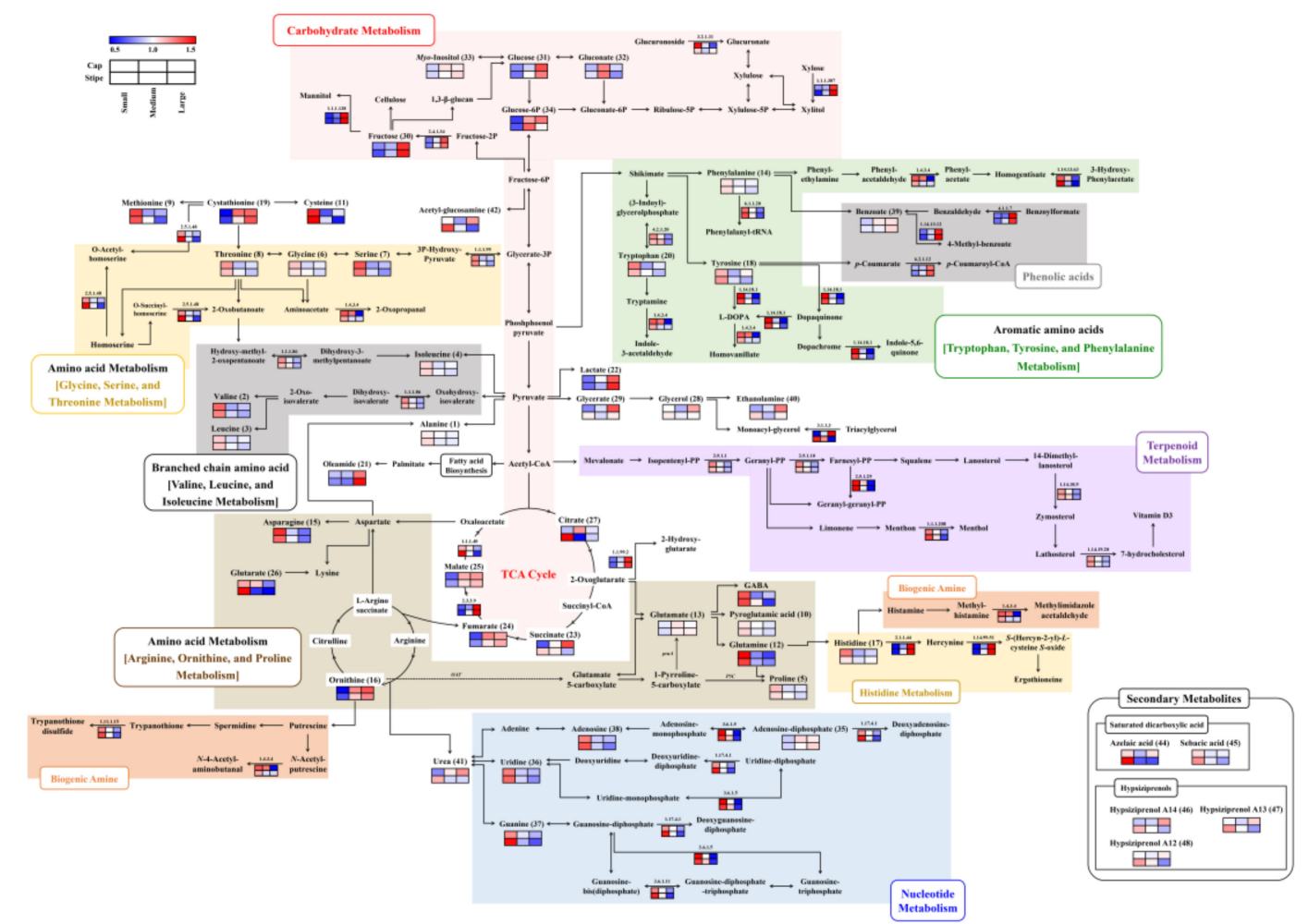

Figure 3. Constructed metabolic pathway that integrated relative metabolite contents and gene expression for three different sizes (small, medium and large) of beech mushrooms. The pathway was modified from the KEGG database (http://www.genome.jp/kegg/). The colored squares (blue-to-red) represent fold changes normalized by the average of each metabolite and gene expression level. EC numbers for the enzymes are listed in Table 3.

Categorically, in the case of small-sized beech mushrooms, most of the amino acids, nucleotides, terpenoids, steroids, and biogenic amines were significantly abundant with higher expression of corresponding genes for both the cap and stipe portions (Figure 3 and Tables 1 and 3 ). In particular, the levels of cysteine, glutamine, and asparagine were approximately 1.5-2-fold higher. The amino acid metabolism-related DEGs displayed a pattern similar to that of the metabolites themselves, but the histidine-derived ergothioneine-related gene, which is a meiotically up-regulated gene 158 protein (EC 2.1.1.44 and 1.14.99.51), exhibited the opposite pattern where it was highly expressed in the 
medium- and large-sized mushrooms. In case of nucleotide metabolism, levels of uridine, guanine, and adenosine decreased according to the size of the mushrooms, while adenosine-diphosphate levels increased. In congruence, the higher mRNA abundance was evident in both the cap and stipe of small-sized mushrooms. Intriguingly, the terpenoid, steroid-, and biogenic amine-related genes were highly expressed in small-sized mushrooms, but their corresponding metabolites were not detected in our data.

In the medium and large-sized mushrooms, most of the carbohydrates and phenolic acid derived metabolites and corresponding DEGs were significantly higher. Notably, the relative abundance of fructose and glucose were significantly higher than those observed in both parts of the small-sized mushrooms. Further, their related genes, except for $\beta$-glucuronidase (EC 3.2.1.31), were approximately 1.5-2-fold higher compared to their expression levels in both parts of the small-sized samples. Additionally, $\beta$-glucan-related genes, including putative $\beta$-glucan synthesis-associated protein (KPE6) and 1,3- $\beta$-glucan synthase (EC 2.4.1.13), showed positive correlation with $\beta$-glucan contents, and this pattern considerably increased in both parts with increasing mushroom sizes (Figure 1B). Considering the metabolite intermediates of TCA cycle, the relative abundance of lactic acid, fumaric acid, and malic acid in both parts of medium- and large-sized beech mushrooms were higher. However, the citric acid and glutaric acid levels in the stipe portion of the small-sized mushrooms were significantly higher than medium and large-sized samples. Further, the phenolic acid-derived metabolites, such as benzoic acid and associated genes including benzoylformate decarboxylase (EC 4.1.1.7), benzoate 4-monooxygenase (EC 1.14.13.12), and 4-coumarate CoA ligase-like 7 (EC 6.2.1.12), were synergistically higher expressed in the medium and large sized mushrooms. In contrast, the levels of certain metabolites exhibited different patterns between the cap and stipe portions in different sized mushrooms. Most notably, the relative abundance of glyceric acid, glycerol, and ethanolamine, and the associated transcript for lipase 4 (EC 3.1.1.3) regulating the glycerolipid metabolism were higher in medium and large-sized mushroom caps, unlike the stipe portions. Additionally, succinic acid and some miscellaneous metabolites including urea, acetyl-glucosamine and hypiziprenols also displayed varying levels depending on different mushroom sizes across their spatial parts (cap and stipe).

\section{Discussion}

Considering the nutritional aspects, beech mushrooms are composed of various amino acids, organic acids, carbohydrates, nucleotides, and secondary metabolites, including hypsiziprenols and terpenoids (Figure 3). The untargeted metabolomic and transcriptomic analyses unraveled the compositional disparity among the different sized beech mushroom (small, medium, and large) samples having various implications.

In small-sized beech mushrooms (cap and stipe), most amino acids, nucleotides, terpenoids, and biogenic amines with corresponding DEGs displayed significantly higher abundance compared to medium- and large-sized mushrooms (Figure 3). Generally, amino acids are directly or indirectly related to the TCA cycle, glycolysis, and the urea cycle in an organism. Most amino acids are crucial metabolites that play an important function in fruiting body development and protein synthesis in mushrooms $[16,17]$. Considering the branched chain amino acid (BCAA) metabolism, higher relative levels of amino acids including valine, isoleucine, and leucine intertwined with higher expression of ketol-acid reductoisomerase (EC 1.1.1.86) were evident in small-sized beech mushrooms compared to medium- or large-sized analogs. BCAAs are crucial precursors for protein synthesis and, in particular, leucine is known to function in the regulation of intracellular signal transduction to control mRNA translation [17]. The serine component of glycine, serine, and threonine metabolism is not only the starting compound for the synthesis of various metabolites such as glycine, cysteine, and serine phospholipids, but is also used as a building block for protein synthesis [18]. The proline biosynthesis pathway from ornithine is also important in mushroom growth. Wagemaker et al. (2007) reported that ornithine can be converted into proline by ornithine aminotransferase (OAT) and pyrroline-5-carboxylate (P5C) and the mRNA of oat exhibit low levels during fruiting body 
developmental stages followed by a slight in transcription from post-harvest developmental stages of mushrooms [19]. In our study, gene expressions for OAT or P5C were not detected, however, the relative levels of proline were high in small-sized beech mushrooms whereas ornithine levels were high in medium and large-sized mushrooms. Proline plays a role in transporting nutrients from the mycelium to fruiting bodies depending on osmotic pressure. Reduction in proline levels prevents the constant outflow of resources from the mycelia to senescent sporocarps, whereas ornithine levels exhibit an increasing trend during growth [19]. Hence, we conjecture that differential expression of genes and metabolite profiles are complementary to the respective growth stages in mushroom development.

Regarding the nucleotide metabolism, the gene expression of ribonucleoside-diphosphate reductase (EC 1.17.4.1), putative exopolyphosphatase (EC 3.6.1.11) and Golgi apyrase (EC 3.6.1.5) were highly up-regulated in small-sized mushrooms. Additionally, metabolite levels of adenosine, guanine, and uridine except adenosine-diphosphate were relatively abundant in both parts (cap and stipe) of small-sized mushrooms compared to their relative levels in the medium- and large-sized mushrooms. Reportedly, nucleotide levels in immature mushrooms are higher than those in the mature mushrooms [20]. Nucleosides are precursor compounds for synthesizing genomic material, regulates reproductive functions, and also serve as energy source within the developing cells in mushrooms $[20,21]$. A similar research study indicated that the levels of nucleotides in the immature mushrooms were higher than those in the mature mushrooms [20].

Terpenoid metabolism involves conversion of farnesyl PP (FPP) to squalene, followed by its multistep enzymatic (farnesyl pyrophosphate synthase, geranylgeranyl diphosphate synthase, and short chain isoprenyl diphosphate synthase) conversion to lanosterol. Lanosterol is further metabolized into triterpenoids, which represent the basic structure of all steroids [22]. We observed the higher relative DEGs involved in terpenoid backbone biosynthesis (EC 2.5.1.1, 2.5.1.10, and 2.5.1.29) and steroid biosynthesis (EC 1.14.19.20 and 1.14.18.9) in small-sized beech mushrooms, however, the related metabolites were not detected in metabolomic profiling. Sterols are important for vegetative growth of fungi as they are the main components of membrane and precursors of steroid hormones regulating the sexual reproduction in mushrooms [22]. Hence, we argue that terpenoid metabolism may be one of the active metabolic pathways required at the early stage of beech mushroom development.

In the case of medium- and large-sized beech mushrooms, a higher abundance of the metabolites related to TCA cycle, carbohydrate metabolism, and phenolic acid metabolism coupled with higher gene levels were observed compared to those in small-sized mushrooms (Figure 3). Generally, the TCA cycle is a series of chemical reactions that occur through the oxidation of acetyl-CoA derived from carbohydrates, fats, and proteins to provide energy. Previously, it has been reported that the levels of organic acids including lactic acid, malic acid, and fumaric acid were higher in the mature stage of mushrooms [23]. The TCA cycle-related metabolites malic acid and fumaric acid perform energy storage functions during the development of the fruiting body [24]. In particular, malate synthase (MS) is the key enzyme of the TCA cycle, and this enzyme contributes to fruiting body formation and is up-regulated during fruiting body development $[25,26]$. Similarly, a higher abundance of carbohydrate derived metabolites including $\beta$-glucans, myo-inositol, glucose, fructose, and glucose-phosphate and their corresponding DEGs were observed for medium- and large-sized mushrooms. Functionally, carbohydrate metabolism influences a number of processes including glycolysis, the TCA cycle, hexose metabolism, and expansion of the fruiting-body in mushrooms besides generating the energy $[10,27,28]$. In particular, myo-inositol is a structural component of the cell membrane, while mannitol functions to promote expansion of the mushroom's fruiting body with its levels heightened during the mature stages [29]. The bioactive phenolic compounds commonly reported in mushrooms are $p$-hydroxybenzoic, $p$-coumaric, and cinnamic acid which protect mushrooms against environmental and oxidative stress [30]. Previously, higher levels of total phenolic contents and several phenolic compounds were reported for matured stage mushrooms [31,32]. In congruence, we substantiated the higher levels of DEGs encoding benzoylformate decarboxylase (EC 4.1.1.7), benzoate 
4-monooxygenase (EC 1.14.13.12) and 4-coumarate CoA ligase-like 7 (EC 6.2.1.12), in large-sized beech mushrooms representing the later stages of development.

Delineating the metabolomic-transcriptomic data across the cap and stipe portions of beech mushrooms, we observed a similar pattern with marginal disparity between spatial parts. Exceptionally, some metabolites including urea, glycerol, acetyl-glucosamine, and hepsiziprenols were differentially expressed in the different parts of beech mushrooms. Biochemically, urea is formed by purine degradation in ornithine cycle and functions the translocation of water and nutrients required for fruiting body development [33]. Wagemaker et al. (2006) reported the higher urease expression in stipe portion which gradually decrease during the later stages of mushroom development [34]. The mushrooms initially grow stipes and then grow caps [34]. In agreement, we observed that glycerol and acetyl-glucosamine, which are the backbone of cell membranes and cell walls, display relatively higher abundance in the stipes (small-sized) and caps (large-sized) of beech mushrooms. Additionally, hypsiziprenols have been poorly characterized in terms of their biosynthetic pathway, but given the general characteristics of secondary metabolites, hypsiziprenols are expected to crucial function in beech mushroom essential for survival and growth in competitive environments [3].

The commercial value of mushroom depends upon its organoleptic and sensory characteristics which in turn maneuvered by its blend of non-volatile and volatile metabolite components. Non-volatile tastant compounds in beech mushrooms mainly include amino acids, carbohydrates, and nucleotides. The hydrophobic amino acids such as proline, glycine, alanine, valine, leucine, tyrosine, and phenylalanine mainly impart the characteristic bitter taste [35]. In particular, proline is a major contributor to the bitter taste as it structurally interacts with respective retronasal receptors [35]. Conversely, glutamic acid, which belongs to the family of mono-sodium glutamate (MSG)-like amino acids, is a well-known contributor to umami taste [35]. In addition, certain nucleotides including $5^{\prime}$-nucleotides (5'-GMP, 5'-IMP, and 5'-GMP), contribute to the umami taste in mushroom [20]. Sugars and sugar alcohols, such as fructose and glucose, are also known to affect sweetness $[10,36]$. Considering the gustatory properties of metabolites, the small-sized beech mushrooms with higher relative abundance of most amino acids and nucleotides might be characterized to have more bitter-umami taste. On the other hand, the higher glutamic acid and carbohydrate sugar contents in large sized mushrooms may affect the more sweet-umami and low bitter taste.

In conclusion, integrated metabolomic and transcriptomic analyses of beech mushroom revealed discriminant metabolic states for its three-different sized (small, medium, and large) samples. Based on our data, we argued that beech mushrooms develop fruiting bodies from mycelium through up-regulating the amino acid, nucleotide, and terpenoid metabolism when they are small or at early stage of development. However, the later stages of fruiting body development involve an up-regulation of carbohydrate metabolism and the TCA cycle. The disparity of metabolite compositions in different sized beech mushrooms may significantly influence their nutritional, functional, gustatory, and sensory characteristics. Our results may aid in delineating the growth process, nutrient composition, and efficient cultivation periods for beech mushrooms based on their subtle metabolite and gene expression profiles.

\section{Materials and Methods}

\subsection{Chemicals and Reagents}

Methanol, acetonitrile, water, and hexane were purchased from Fisher Scientific (Pittsburgh, PA, USA). Methoxyamine hydrochloride, pyridine, $N$-methyl- $N$-(trimethylsilyl)-trifluoroacetamide (MSTFA), glacial acetic acid, and sulfuric acid were obtained from Sigma Chemical Co. (St. Louis, MO, USA). Sodium hydroxide and potassium hydroxide were purchased from Junsei Chemical (Tokyo, Japan). All enzymes and reagent buffers used were purchased from Megazyme Ltd. (Wicklow, Ireland). 


\subsection{Mushroom Materials}

Brown (KMCC03087 and KMCC03109) and white (KMCC03106, and KMCC03108) beech mushroom fruiting bodies were provided by Bumwoo mushroom farm (Yeoju-si, Gyeonggi-do, Korea). The samples were cultivated using bottle technology for about three months, with four bottles of each strains were procured. Photographs of the fruiting body samples are shown in Figure 1B. The beech mushroom samples were randomly classified based morphology into three different sizes namely small, medium, and large. According to the diameter of cap portions, mushrooms were classified into small $(<1.0 \mathrm{~cm})$, medium (between 1.0 and $2.0 \mathrm{~cm})$, and large $(>2.0 \mathrm{~cm})$, respectively. Growth characteristics of beech mushroom samples of different sizes are described in Table S1. Further, the fruiting bodies were separated into the cap and stipe portions prior to analyses. Three biological replications of four strains were used in this study.

\subsection{Sample Preparation for Metabolite Profiling}

The fruiting body samples were ground using liquid nitrogen and immediately placed in microfuge tubes. The number of fruiting bodies (cap and stipe portions) used for grinding were 10, 5, and 2 for small, medium, and large sizes, respectively. Ground samples $(1 \mathrm{~g})$ were extracted with $1 \mathrm{~mL}$ of $80 \%$ methanol by sonication for $5 \mathrm{~min}$ at $4{ }^{\circ} \mathrm{C}$ (Hettich Zentrifugen Universal 320, Tuttlingen, Germany) and then homogenized using a Retsch MM400 Mixer Mill (Retsch GmbH and Co., Haan, Germany) at $30 \mathrm{~Hz}$ for $10 \mathrm{~min}$. After centrifugation for $10 \mathrm{~min}$ at 12,000 rpm and $4{ }^{\circ} \mathrm{C}$ (Gyrozen 1730R, Gyrozen Inc., Daejeon, Korea), the supernatants were filtered through a $0.2 \mu \mathrm{m}$ polytetrafluoroethylene (PTFE) filter (Chromdisc, Daegu, Korea) and completely dried using a speed-vacuum concentrator (Biotron, Seoul, Korea). Dried extracts were re-dissolved in $80 \%$ methanol to a final concentration of $10 \mathrm{mg} / \mathrm{mL}$ for gas chromatography-time of flight-mass spectrometry (GC-TOF-MS) and ultrahigh performance liquid chromatography-linear trap quadruple-ion trap-mass spectrometry/mass spectrometry (UHPLC-LTQ-IT-MS/MS) analyses. For GC-TOF-MS analysis, the re-dissolved samples were completely dried again using a speed-vacuum concentrator prior to derivatization step. First, the dried samples were oximated with $50 \mu \mathrm{L}$ of methoxyamine hydrochloride (20 mg/mL in pyridine) at $30^{\circ} \mathrm{C}$ for $90 \mathrm{~min}$. Next, $50 \mu \mathrm{L}$ of the MSTFA was added to the samples for $30 \mathrm{~min}$ at $37^{\circ} \mathrm{C}$.

\subsection{RNA Isolation for Transcriptome Analyis}

RNA extraction was performed according to previous study [37]. The number of different-sized mushroom portions (cap and stipe) and biological replicates $(n=3)$ used for RNA extraction was the same as for metabolite extraction. The fruiting body of beech mushrooms were homogenized in liquid nitrogen with a mortar and pestle. The resulting homogenate was aliquoted to $1 \mathrm{~g}$ in Eppendorf-tubes and stored at $-70{ }^{\circ} \mathrm{C}$. Total RNA was extracted from each sample of the leaves separately using an RNeasy plant mini Kit (Qiagen, Valencia, CA, USA) according to the manufacturer's instructions. RNA samples from H. marmoreus of different sizes were sequenced using an Illumina Hiseq2000 system, and 112,133,856 total reads were generated. The raw sequence data were extracted for the base pairs using the SolexaQA package. All sequence reads obtained from the samples of different sizes to optimize de novo assembly were used to assess $k$-mer sizes and assembled contigs. To select the best de novo assembly, several hash lengths were considered. The transcript annotation of $H$. marmoreus was defined by de novo assembly and validated by comparison to amino acid sequences in NCBI NR fungi, Uniprotkb fungi, KOG, and the KEGG database using BLASTX (e-value $\leq 1 \times 10^{-10}$ ). The reads for each sequence tag were mapped to the assembled transcripts using Bowtie 2 (Johns Hopkins University, Baltimore, MD, USA, http://bowtie-bio.sourceforge.net) software (mismatch $\leq 2 \mathrm{bp}$ ), and the number of mapped clean reads for each transcript were counted. Then, mapped clean reads were normalized using the DESeq library as an R/Bioconductor package. To select differentially expressed genes (DEGs) among each analyzed sample, a two-fold change method to identify differences in expression that were 
above two-fold and a binomial test to satisfy the FDR ( $p$-value) as less than 0.01 were simultaneously applied and calculated via DESeq. The process of RNA sequence analysis (de novo assembly, ordering contigs, and annotation) was performed by SEEDERS Company (Daejeon, South Korea).

\subsection{GC-TOF-MS Analysis and UHPLC-LTQ-IT-MS/MS Analysis}

GC-TOF-MS analysis was performed according to our previous study [3]. An Agilent 7890 gas chromatography, coupled with an Agilent 7693 autosampler (Agilent, Santa Clara, CA, USA) and installed with a Pegasus high-throughput (HT)-TOF-MS (Leco Corp., St. Joseph, MI, USA) program, was used for GC-TOF-MS analysis. Metabolites were separated by an Rtx-5MS column (30 m inner diameter $\times 0.25 \mathrm{~mm}$ i.d.; $0.25 \mathrm{~mm}$ particle size; Restek Corp. Bellefonte, PA, USA) was used with helium as the carrier gas at a constant flow rate of $1.5 \mathrm{~mL} / \mathrm{min}$. The GC oven temperature was maintained at $75^{\circ} \mathrm{C}$ for $2 \mathrm{~min}$, increased to $300^{\circ} \mathrm{C}$ as final temperature over $15 \mathrm{~min}$ at rate of $15^{\circ} \mathrm{C} / \mathrm{min}$. The front inlet and transfer line temperatures were $250^{\circ} \mathrm{C}$ and $240^{\circ} \mathrm{C}$, respectively. The scanning mass range was $50-1000 \mathrm{~m} / \mathrm{z}$ and the detector voltage was $1650 \mathrm{~V}$. The derivatized samples $(1 \mu \mathrm{L})$ were injected into a GC-TOF-MS instrument under split ratio (1:5). UHPLC-LTQ-IT-MS/MS was performed according to the method adapted from Park et al. [3] on the LTQ-XL mass spectrometry installed with ion trap-electrospray interface (Thermo Fisher Scientific, San José, CA) coupled with RS Autosampler, DIONEX UltiMate 3000 RS Pump, RS Column Compartment and RS Diode Array Detector (Dionex Corporation, Sunnyvale, CA, USA). Mushroom samples were separated on a Thermo Scientific Syncronis C18 UHPLC column with a $1.7 \mu \mathrm{m}$ particle size. The flow rate was maintained at $0.3 \mathrm{~mL} / \mathrm{min}$ with a $10 \mu \mathrm{L}$ injection volume. The mobile phase consisted of Solvent A $(0.1 \%$ formic acid in water) and Solvent B ( $0.1 \%$ formic acid in acetonitrile), the gradient flow was programmed as follow: The initial solvent condition was $10 \%$ of solvent $B$; the gradient was steadily increased from $10 \%$ solvent B to $100 \%$ solvent B over $18 \mathrm{~min}$. Then, decreased to the initial conditions (10\% solvent B) over $4 \mathrm{~min}$, completing the total run time of $22 \mathrm{~min}$. Mass spectra were obtained by electrospray ionization in positive and negative ion mode within a mass range of 150-1000 m/z. The photodiode array detector was programed at a wavelength range of $200-600 \mathrm{~nm}$ for detection and managed by $3 \mathrm{D}$ field. The operational parameters were as follows: Source voltage, $\pm 5 \mathrm{kV}$; capillary voltage, $39 \mathrm{~V}$; capillary temperature, $275^{\circ} \mathrm{C}$. Tandem MS analysis was carried out scan-type turbo data-dependent scanning under the conditions used for MS scanning. The analytical samples were randomly analyzed in blocks of ten runs followed by intermitted quality control (QC) samples to reduce systematic errors.

\subsection{Measurement of $\beta$-Glucan Contents}

The $\beta$-glucan contents were determined using a glucan assay kit (Megazyme Ltd., Wicklow, Ireland) according to the manufacturer's procedures and our previous study [3], with some modification. For measurement of the total ( $\alpha$ - and $\beta$-) glucan contents, the 24 dried beech mushroom samples (90 mg) were milled using a mortar and pestle, and $2 \mathrm{~mL}$ of cold $12 \mathrm{M}$ sulfuric acid was added. Then, $10 \mathrm{~mL}$ of distilled water was added to each sample, and the samples were incubated in a boiling water bath at $100{ }^{\circ} \mathrm{C}$ for $2 \mathrm{~h}$. After boiling, the samples were neutralized with $10 \mathrm{M} \mathrm{KOH}$, where $200 \mathrm{mM}$ (pH 5.0) was added for a total volume of $100 \mathrm{~mL}$. After centrifugation $\left(1500 \times \mathrm{g}\right.$ ) for $15 \mathrm{~min}$ at $4{ }^{\circ} \mathrm{C}$ (Universal $320 \mathrm{R}$, Hettich, Zentrifugen, Germany), the reaction aliquots $(100 \mu \mathrm{L})$ were mixed with $100 \mu \mathrm{L}$ of exo-1,3- $\beta$-glucanase $(20 \mathrm{U} / \mathrm{mL})$ and $\beta$-glucosinase $(4 \mathrm{U} / \mathrm{mL})$ in $200 \mathrm{mM}$ sodium acetate buffer (pH 5.0) and incubated at $40^{\circ} \mathrm{C}$ for $1 \mathrm{~h}$. Next, $3 \mathrm{~mL}$ of glucose determination reagent (GOPOD) was added and incubated at $40^{\circ} \mathrm{C}$ for $20 \mathrm{~min}$. The absorbance was recorded for the reacted samples at $510 \mathrm{~nm}$.

For the determination of the $\alpha$-glucan (starch and phytoglycogen) contents, the dried mushroom samples (100 mg) were dissolved in $2 \mathrm{~mL}$ of $2 \mathrm{M} \mathrm{KOH}$ and subsequently added to each sample with $8 \mathrm{~mL}$ of $1.2 \mathrm{M}$ sodium acetate buffer ( $\mathrm{pH} 3.8)$. Then, amyloglucosidase $(1630 \mathrm{U} / \mathrm{mL})$ and invertase $\left(500 \mathrm{U} / \mathrm{mL}\right.$ ) were added to the solution and the samples were incubated at $40{ }^{\circ} \mathrm{C}$ for $20 \mathrm{~min}$. After centrifugation $\left(1500 \times \mathrm{g}\right.$ ) for $15 \mathrm{~min}$ at $4{ }^{\circ} \mathrm{C}$ (Universal $320 \mathrm{R}$, Hettich, Zentrifugen, Germany), $100 \mu \mathrm{L}$ 
of the aliquot was mixed with $100 \mu \mathrm{L}$ of $200 \mathrm{mM}$ sodium acetate buffer (pH 5.0) and $3 \mathrm{~mL}$ of GOPOD reagent followed by an incubation at $40{ }^{\circ} \mathrm{C}$ for $20 \mathrm{~min}$. The absorbance was recorded for the reacted samples at $510 \mathrm{~nm}$. The levels of $\beta$-glucan were measured by subtracting $\alpha$-glucan from the total glucan contents.

\subsection{Data Processing and Multivariate Statistical Analysis}

The GC-TOF-MS and UHPLC-LTQ-IT-MS/MS raw data files were converted to NetCDF ( ${ }^{*}$.cdf) format using Leco ChromaTOF software (Version 4.44) and Thermo Xcalibur software (version 2.1, Thermo Fisher Scientific). After conversion, the alignments of NetCDF files were processed by the MetAlign software package (http://www.metalign.nl) to obtain data including retention times (min), normalized peak intensities, and corresponding mass $(\mathrm{m} / \mathrm{z})$. The MetAlign parameters were as follows: Peak slope factor, 2.0; peak threshold factor, 3.0; peak threshold, 10; average peak width at half height, 40, which correspond to a mass range of 50-1000 for GC-MS. In case of LC-MS, a peak threshold of 10 and average peak width at half height of 70 were set. The aligned data were applied to a treatment process for dealing with missing values and transformed to a data set for classification. The resulting data were exported to an Excel file, and statistical analysis was performed using SIMCA-P+ software (version 12.0, Umetrics, Umea, Sweden) to compare metabolites among samples. The data sets were auto-scaled (unit variance scaling), log-transformed and mean-centered in a column-wise fashion prior to principal component analysis (PCA) and partial least squares discriminant analysis (PLS-DA).

Principal component analysis (PCA), partial least-squares discriminant analysis (PLS-DA) and orthogonal partial least-squares discriminant analysis (OPLS-DA) were used to compare the different metabolites of the samples. Analysis of variance testing of cross-validated predictive residuals (CV-ANOVA) is a diagnostic tool for assessing the reliability of PLS and OPLS models applied by SIMCA-P+ version 12.0. CV-ANOVA uses a significance test (F-test) of the null hypothesis of equal residuals of the two compared models. The F-test, based on the ratio MS regression/MS residual, analyzes the significance of model [38]. The model of PLS-DA and OPLS-DA based on our MS data showed a significantly different model $(p$-value $<0.05)$. The significantly discriminant metabolites were selected based on the variable importance in the projection (VIP) values $>0.7$ and tested for significance at $p$-value $<0.05$. The selected metabolites obtained from GC-TOF-MS and UHPLC-LTQ-IT-MS/MS were tentatively identified based on various data comparing their retention time, mass spectrum, $\mathrm{MS}^{\mathrm{n}}$ fragment, and UV spectrum data with those for standard compounds analyzed under identical condition and various available database including published papers [3], the National Institute of Standards and Technology (NIST) database (Version 2.0, 2011, FairCom, Gaithersburg, MD, USA), the Human Metabolome Database (HMDB; http://www.hmdb.ca/), Wiley 9, In house libraries, and Dictionary of Natural Product (CCD, Copyright 2008, Taylor and Francis Group, Boca Raton, FL, USA). Significant statistical differences including metabolome and RNA-sequencing data were determined by analysis of variance (ANOVA) coupled with Duncan's multiple range tests using PASW statistica 18 (SPAA, Inc., Chicago, IL, USA) and visualized by heat map analysis using MEV software, version 4.8 (multiple array viewer).

Supplementary Materials: Supplementary materials can be found at http://www.mdpi.com/1422-0067/20/23/ 6007/s1.

Author Contributions: Conceptualization: C.H.L. and J.-G.K.; validation: S.Y.S, and E.S.J.; formal analysis: Y.J.P.; investigation: S.Y.S. and Y.J.P.; resources: Y.W.L.; writing—original draft preparation: S.Y.S.; writing—review and editing: E.S.J. and D.S.; supervision: C.H.L. and J.-G.K; funding acquisition: E.S.J. and J.K.

Acknowledgments: This work was supported by a grant from the Next-Generation BioGreen 21 Program grant no. PJ01334603 and PJ01044002, Rural Development Administration, Republic of Korea.

Conflicts of Interest: The authors declare no conflict of interest. 


\section{Abbreviations}

\section{GC-TOF-MS}

\section{UHPLC-LTQ-IT-MS/MS}

PCA

PLS-DA

OPLS-DA

DEG

EC

\author{
Gas chromatography-time of flight-mass spectrometry \\ Ultrahigh performance liquid chromatography-linear trap \\ quadruple-ion trap-mass spectrometry/mass spectrometry \\ Principal component analysis \\ Partial least squares-discriminant analysis \\ Orthogonal partial least squares-discriminant analysis \\ Differentially expressed gene \\ Enzyme commission
}

\section{References}

1. Cho, I.H.; Kim, Y.S.; Choi, H.K. Metabolomic discrimination of different grades of pine-mushroom (Tricholoma matsutake Sing.) using ${ }^{1} \mathrm{H}$ NMR spectrometry and multivariate data analysis. J. Pharm. Biomed. Anal. 2007, 43, 900-904. [CrossRef]

2. Politowicz, J.; Lech, K.; Lipan, L.; Figiel, A.; Carbonell-Barrachina, Á.A. Volatile composition and sensory profile of shiitake mushrooms as affected by drying method. J. Sci. Food Agric. 2018, 98, 1511-1521. [CrossRef]

3. Park, Y.J.; Jung, E.S.; Singh, D.; Lee, D.E.; Kim, S.; Lee, Y.W.; Kim, J.G.; Lee, C.H. Spatial (cap \& stipe) metabolomic variations affect functional components between brown and white beech mushrooms. Food Res. Int. 2017, 102, 544-552. [CrossRef]

4. Harada, A.; Gisusi, S.; Yoneyama, S.; Aoyama, M. Effects of strain and cultivation medium on the chemical composition of the taste components in fruit-body of Hypsizygus marmoreus. Food Chem. 2004, 84, 265-270. [CrossRef]

5. Sun, S.; Li, X.; Ruan, L.; Zhang, L.; Hu, K. A novel breeding strategy for new strains of Hypsizygus marmoreus and Grifola frondosa based on ligninolytic enzymes. World J. Microbiol. Biotechnol. 2014, 30, 2005-2013. [CrossRef] [PubMed]

6. Lee, S.H.; Kim, M.K.; Lee, M.K.; Kim, N.R.; Lee, C.Y.; Lee, H.S. Electrophoretic karyotyping of Hypsizygus marmoreus and evaluation of variation among its basidiospores. FEMS Microbiol. Lett. 2014, 359, 209-215. [CrossRef] [PubMed]

7. Lee, S.; Do, S.G.; Kim, S.Y.; Kim, J.; Jin, Y.; Lee, C.H. Mass spectrometry-based metabolite profiling and antioxidant activity of Aloe vera (Aloe barbadensis Miller) in different growth stages. J. Agric. Food Chem. 2012, 60, 11222-11228. [CrossRef] [PubMed]

8. Jang, Y.K.; Jung, E.S.; Lee, H.A.; Choi, D.; Lee, C.H. Metabolomic characterization of hot pepper (Capsicum annuum "CM334") during fruit development. J. Agric. Food Chem. 2015, 63, 9452-9460. [CrossRef]

9. Lee, H.J.; Suh, D.H.; Jung, E.S.; Park, H.M.; Jung, G.Y.; Do, S.G.; Lee, C.H. Metabolomics of Lonicera caerulea fruit during ripening and its relationship with color and antioxidant activity. Food Res. Int. 2015, 78, 343-351. [CrossRef]

10. Harada, A.; Yoneyama, S.; Doi, S.; Aoyama, M. Changes in contents of free amino acids and soluble carbohydrates during fruit-body development of Hypsizygus marmoreus. Food Chem. 2003, 83, 343-347. [CrossRef]

11. Hirai, M.Y.; Klein, M.; Fujikawa, Y.; Yano, M.; Goodenowe, D.B.; Yamazaki, Y.; Kanaya, S.; Nakamura, Y.; Kitayama, M.; Suzuki, H.; et al. Elucidation of gene-to-gene and metabolite-to-gene networks in Arabidopsis by integration of metabolomics and transcriptomics. J. Biol. Chem. 2005, 280, 25590-25595. [CrossRef] [PubMed]

12. Gao, W.; Sun, H.X.; Xiao, H.; Cui, G.; Hillwig, M.L.; Jackson, A.; Wang, X.J.; Shen, Y.; Zhao, N.; Zhang, L.; et al. Combining metabolomics and transcriptomics to characterize tanshinone biosynthesis in Salvia miltiorrhiza. BMC Genom. 2014, 15, 73. [CrossRef] [PubMed]

13. Kim, H.Y.; Heo, D.Y.; Park, H.M.; Singh, D.; Lee, C.H. Metabolomic and Transcriptomic Comparison of Solid-State and Submerged fermentation of Penicillium expansum KACC 40815. PLoS ONE 2016, 11, e0149012. [CrossRef] [PubMed]

14. Huang, H.; Yao, Q.; Xia, E.; Gao, L. Metabolomics and Transcriptomics Analyses Reveal Nitrogen Influences on the Accumulation of Flavonoids and Amino Acids in Young Shoots of Tea Plant (Camellia sinensis L.) Associated with Tea Flavor. J. Agric. Food Chem. 2018, 66, 9828-9838. [CrossRef] [PubMed] 
15. Viant, M.R.; Kurland, I.J.; Jones, M.R.; Dunn, W.B. How close are we to complete annotation of metabolomes? Curr. Opin. Chem. Biol. 2017, 36, 64-69. [CrossRef]

16. Moore, D.; Gange, A.C.; Gange, E.G.; Boddy, L. Fruit bodies: Their production and development in relation to environment. In Ecology of Saprotrophic Basidiomycetes, 1st ed.; Boddy, L., Frankland, J.C., van West, P., Eds.; Elsevier Academic Press: Amsterdam, Netherlands, 2008; Volume 28.

17. Liu, X.; Wang, J.; Xu, J.; Shi, J. FgIlv5 is required for branched-chain amino acid biosynthesis and full virulence in Fusarium graminearum. Microbiology 2014, 160, 692-702. [CrossRef] [PubMed]

18. Shimizu, Y.; Sakuraba, H.; Doi, K.; Ohshima, T. Molecular and functional characterization of D-3-phosphoglycerate dehydrogenase in the serine biosynthetic pathway of the hyperthermophilic archaeon Sulfolobus tokodaii. Arch. Biochem. Biophys. 2008, 470, 120-128. [CrossRef]

19. Wagemaker, M.J.M.; Eastwood, D.C.; Welagen, J.; Van der Drift, C.; Jetten, M.S.M.; Burton, K.; Van Griensven, L.J.L.D.; Op Den Camp, H.J.O. The role of ornithine aminotransferase in fruiting body formation of the mushroom Agaricus bisporus. Mycol. Res. 2007, 111, 909-918. [CrossRef]

20. Phan, C.W.; Wang, J.K.; Cheah, S.C.; Naidu, M.; David, P.; Sabaratnam, V. A review on the nucleic acid constituents in mushrooms: Nucleobases, nucleosides and nucleotides. Crit. Rev. Biotechnol. 2018, 38, 762-777. [CrossRef]

21. Rahi, D.K.; Malik, D. Diversity of mushrooms and their metabolites of nutraceutical and therapeutic significance. J. Mycol. 2016, 2016, 1-18. [CrossRef]

22. Wang, M.; Gu, B.; Huang, J.; Jiang, S.; Chen, Y.; Yin, Y.; Pan, Y.; Yu, G.; Li, Y.; Wong, B.H.C.; et al. Transcriptome and proteome exploration to provide a resource for the study of Agrocybe aegerita. PLoS ONE 2013, 8, e56686. [CrossRef] [PubMed]

23. Wang, J.; Li, W.; Li, Z.; Wu, W.; Tang, X. Analysis and evaluation of the characteristic taste components in portobello mushroom. J. Food Sci. 2018, 83, 1542-1551. [CrossRef] [PubMed]

24. Deja, S.; Wieczorek, P.P.; Halama, M.; Jasicka-Misiak, I.; Kafarski, P.; Poliwoda, A.; Młynarz, P. Do differences in chemical composition of stem and cap of Amanita muscaria fruiting bodies correlate with topsoil type? PLoS ONE 2014, 9, e104084. [CrossRef] [PubMed]

25. Yoon, J.J.; Munir, E.; Miyasou, H.; Hattori, T.; Shimada, M.; Terashita, T. A possible role of the key enzymes of the glyoxylate and gluconeogenesis pathways for fruit-body formation of the wood-rotting basidiomycete Flammulina velutipes. Mycoscience 2002, 43, 327-332. [CrossRef]

26. Peraza-Reyes, L.; Berteaux-Lecellier, V. Peroxisomes and sexual development in fungi. Front. Physiol. 2013, 4, 244. [CrossRef]

27. Ceccaroli, P.; Buffalini, M.; Saltarelli, R.; Barbieri, E.; Polidori, E.; Ottonello, S.; Kohler, A.; Tisserant, E.; Martin, F.; Stocchi, V. Genomic profiling of carbohydrate metabolism in the ectomycorrhizal fungus Tuber melanosporum. New Phytol. 2011, 189, 751-764. [CrossRef]

28. Teramoto, M.; Wu, B.; Hogetsu, T. Transfer of 14C-photosynthate to the sporocarp of an ectomycorrhizal fungus Laccaria amethystina. Mycorrhiza 2012, 22, 219-225. [CrossRef]

29. Barros, L.; Baptista, P.; Estevinho, L.M.; Ferreira, I.C. Effect of fruiting body maturity stage on chemical composition and antimicrobial activity of Lactarius sp. mushrooms. J. Agric. Food Chem. 2007, 55, 8766-8771. [CrossRef]

30. Muszyńska, B.; Grzywacz-Kisielewska, A.; Kała, K.; Gdula-Argasińska, J. Anti-inflammatory properties of edible mushrooms: A review. Food Chem. 2018, 243, 373-381. [CrossRef]

31. Soares, A.A.; de Souza, C.G.M.; Daniel, F.M.; Ferrari, G.P.; da Costa, S.M.G.; Peralta, R.M. Antioxidant activity and total phenolic content of Agaricus brasiliensis (Agaricus blazei Murril) in two stages of maturity. Food Chem. 2009, 112, 775-781. [CrossRef]

32. Valdez-Morales, M.; Carlos, L.C.; Valverde,M.E.; Ramírez-Chávez, E.; Paredes-López, O. Phenolic compounds, antioxidant activity and lipid profile of huitlacoche mushroom (Ustilago maydis) produced in several maize genotypes at different stages of development. Plant Food Hum. Nutr. 2016, 71, 436-443. [CrossRef] [PubMed]

33. Wagemaker, M.J.M.; Eastwood, D.C.; van der Drift, C.; Jetten, M.S.M.; Burton, K.; Van Griensven, L.J.L.D.; Op den Camp, H.J.M. Expression of the urease gene of Agaricus bisporus: A tool for studying fruit body formation and post-harvest development. Appl. Microbiol. Biotechnol. 2006, 71, 486-492. [CrossRef] [PubMed]

34. Kües, U.; Liu, Y. Fruiting body production in basidiomycetes. Appl. Microbiol. Biotechnol. 2000, 54, 141-152. [CrossRef] [PubMed] 
35. Zhao, C.J.; Schieber, A.; Gänzle, M.G. Formation of taste-active amino acids, amino acid derivatives and peptides in food fermentations-A review. Food Res. Int. 2016, 89, 39-47. [CrossRef] [PubMed]

36. Aprea, E.; Charles, M.; Endrizzi, I.; Corollaro, M.L.; Betta, E.; Biasioli, F.; Biasioli, F.; Gasperi, F. Sweet taste in apple: The role of sorbitol, individual sugars, organic acids and volatile compounds. Sci. Rep. 2017, 7, 44950. [CrossRef]

37. Min, B.; Kim, S.; Oh, Y.L.; Kong, W.S.; Park, H.; Cho, H.; Jang, K.; Kim, J.; Choi, I.G. Genomic discovery of the hypsin gene and biosynthetic pathways for terpenoids in Hypsizygus marmoreus. BMC Genom. 2018, $19,789$. [CrossRef]

38. Eriksson, L.; Trygg, J.; Wold, S. CV-ANOVA for significance testing of PLS and OPLS ${ }^{\circledR}$ models. J. Chemometr. 2008, 22, 594-600. [CrossRef]

(C) 2019 by the authors. Licensee MDPI, Basel, Switzerland. This article is an open access article distributed under the terms and conditions of the Creative Commons Attribution (CC BY) license (http://creativecommons.org/licenses/by/4.0/). 